\title{
3-D Measurement of Solder Paste Using Two-Step Phase Shift Profilometry
}

\author{
Tak-Wai Hui and Grantham Kwok-Hung Pang, Senior Member, IEEE
}

\begin{abstract}
A two-step phase shift profilometry method (2-step PSP) with prefiltering and postfiltering stages is proposed to reconstruct the 3-D profile of solder paste. Two sinusoidal patterns which are $\pi$-out-of-phase are used in the 3-D reconstruction. The new method uses only two fringe patterns rather than four as the fourstep phase shift profilometry (4-step PSP). In Fourier transform profilometry (FTP), a bandpass filter is required to extract the fundamental spectrum from the background and higher order harmonics due to camera noise and imperfectness of the pattern projector. By using two $\pi$-out-of-phase sinusoidal fringe patterns, the background term can be eliminated directly by taking the average of the two fringe patterns. The fringe pattern which is close to its ideal form can also be recovered from the averaging process. Prefiltering is utilized in filtering raw images to remove noise causing higher order harmonics. Hilbert transform is then used to obtain the in-quadrate component of the processed fringe pattern. Postfiltering is applied for reconstructing an appropriate 3-D profile.
\end{abstract}

Index Terms-3-D measurement, Fourier transform profilometry, Hilbert transform, phase shift profilometry, solder paste.

\section{INTRODUCTION}

$\mathbf{N}$ OWADAYS, surface-mount technology (SMT) is increasing in popularity. The manufacture of printed circuit boards (PCBs) involves a number of steps [1], [2]. First, a bare-board PCB is made. Second, solder paste is printed onto the copper pads of the PCB. Third, electronic components are placed and then pasted by reflow. Fourth, the PCB is cleaned to remove flux. Lastly, the functionality of the PCB is tested (in-circuit test). PCB manufacturing needs quality control at every production stage. This can be achieved via inspection, and provides a feedback mechanism for closed-loop process control.

Due to the use of SMT, the production rate of PCB assembly has significantly increased when compared to through-hole component designs. The component size is continuously shrinking. It is estimated that $52 \%-71 \%$ of SMT defects are related to the solder paste printing process [3], [4] . Among all the production stages, solder paste printing is the most critical step in the PCB production. The deposited solder paste that

Manuscript received October 3, 2007; revised May 8, 2008. Current version published October 3, 2008. This work was recommended for publication by Associate Editor P. Conway upon evaluation of the reviewers comments.

The authors are with the Department of Electrical and Electronic Engineering, University of Hong Kong, Hong Kong, China (e-mail: twhui@eee.hku.hk; eetwhui@gmail.com; gpang@eee.hku.hk).

Color versions of one or more of the figures in this paper are available online at http://ieeexplore.ieee.org.

Digital Object Identifier 10.1109/TEPM.2008.2004573 forms the solder joint provides both mechanical and electrical interconnections for the components [5]. The volume of each printed solder paste block is important because of its impact on solder joint quality and the assembled PCB's subsequent long-term reliability [6], [27].

There are many optical 3-D reconstruction methods for solder paste blocks. In laser triangulation [7], [8], a spot of light from a laser diode is pointed onto the surface of solder paste under inspection. The reflected laser enters into the window of a lightsensitive digital detector array. The height of a point of the solder paste can then be found by triangulation. In practice, a line of laser is projected instead of a laser spot. When the projecting device moves across the targeted object, a camera is used to capture a number of images at even time intervals. If high resolution is required, then more images (and also measurement time) are required. The technique of shape from shading can be applied for reconstructing object with well-defined reflectance model [9], [10]. A complex mathematical model is needed to generate the suitable reflectance model of the object under inspection. The model depends on a lot of parameters. In the real world, it is very difficult to model the reflectance of the object well. Another method is to use artificial neural networks (ANNs) [12]. Once the network is properly trained, it can reconstruct solder paste under inspection. The drawback of ANNs is the requirement of reference samples. ANNs can also be used to inspect solder joints without the need of 3-D reconstruction [11], [13]. Fuzzy logic and ANNs can also be used together in inspecting solder joints. However, both methods cannot provide volume information. Phase profilometry is an attractive 3-D reconstruction method [16]-[23]. It can measure the 3-D profile without the requirement of reference samples. One or more sinusoidal patterns are projected onto the targeted object. Due to the surface variation of the object, the projected pattern will be deformed. Two phase distributions can be formed with and without the presence of the object. The difference of the two phase distributions has a direct relationship with the surface profile of the object. According to the way of extracting the object phase from the deformed pattern, the method of phase profilometry can be classified into two kinds. One is phase-shift profilometry (PSP) and another one is Fourier transform profilometry (FTP). In PSP, multiple phase-shifted patterns are required. For FTP, only one pattern is required.

This paper is organized as follows. Section II reviews the background of four-step phase shift profilometry and Fourier transform profilometry. Section III introduces the proposed twostep phase shift profilometry with prefiltering and postfiltering stages. In Section IV, the performance of the proposed system in terms of volume accuracy and root-mean-square error (RMSE) are evaluated. This paper is concluded in Section V. 


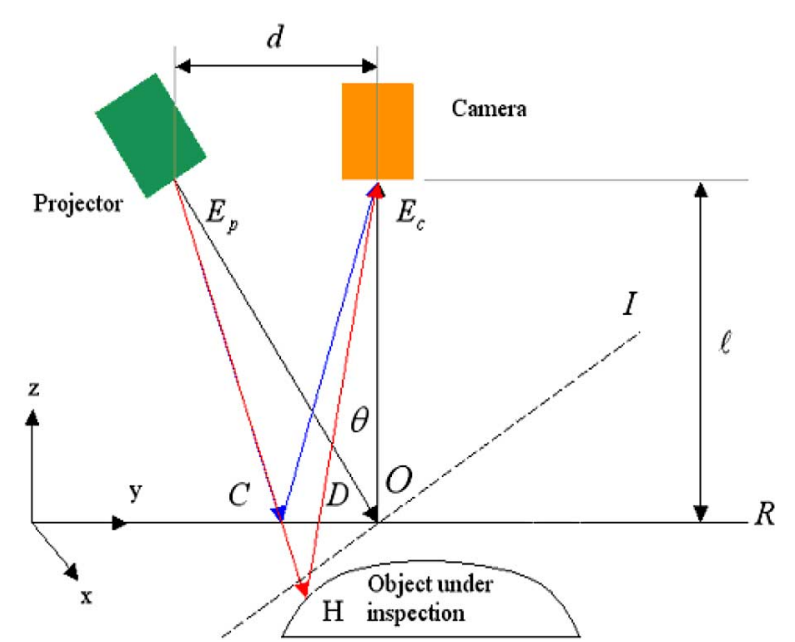

Fig. 1. Light ray diagram of the optical setup.

\section{BACKGROUND}

\section{A. Principle of Four-Step Phase-Shift Profilometry}

The optical geometry of the phase measuring techniques is shown in Fig. 1. There is a virtual plane $I$ which is perpendicular to the optical axis of the projector. $E_{P}$ and $E_{C}$ are the exit pupil of the projector lens and the entrance pupil of the camera lens, respectively. Both of them lie on the same plane such that $d$ is the distance between $E_{P}$ and $E_{C}$. The height $\ell$ is the vertical distance from $E_{C}$ or $E_{P}$ to the reference plane $R$. The angle between the virtual plane $I$ and the reference plane $R$ is $\theta$. The principal light ray $E_{P} O$ forms an angle $\theta$ with respect to $E_{c} O$. Sinusoidal patterns are projected onto the reference plane and the object under inspection from the projector. The resulted images are captured by the camera for further processing.

When there is no object present, a light ray passes through the exit pupil of the projector lens $\left(E_{P}\right)$ and reflects at point $C$ on the reference plane. The captured reference pattern $I_{0 n}(x, y)$ is given by [22]

$I_{0 n}(x, y)=a_{0}(x, y)+b_{0}(x, y) \cos \left[2 \pi f_{0} y+\phi_{0}(x, y)+\frac{n \pi}{2}\right]$

where $\mathrm{n}=0,1,2$ and 3. $a_{0}(x, y), b_{0}(x, y)$, and $f_{0}$ are background intensity, intensity modulation, and fundamental frequency of the projected reference patterns, respectively. $\phi_{0}(x, y)+n \pi / 2$ is the phase modulation.

With the presence of the object, the same light ray reflects at point $H$ on the surface of the object. It passes through point $D$ at the reference plane to the image detector via the entrance of the camera pupil. Similarly, the captured pattern $I_{n}(x, y)$ is given by [22]

$$
I_{n}(x, y)=a(x, y)+b(x, y) \cos \left[2 \pi f_{0} y+\phi(x, y)+\frac{n \pi}{2}\right]
$$

where $\mathrm{n}=0,1,2$ and 3. $a(x, y)$ and $b(x, y)$ are background intensity and intensity modulation respectively. $\phi(x, y)+n \pi / 2$

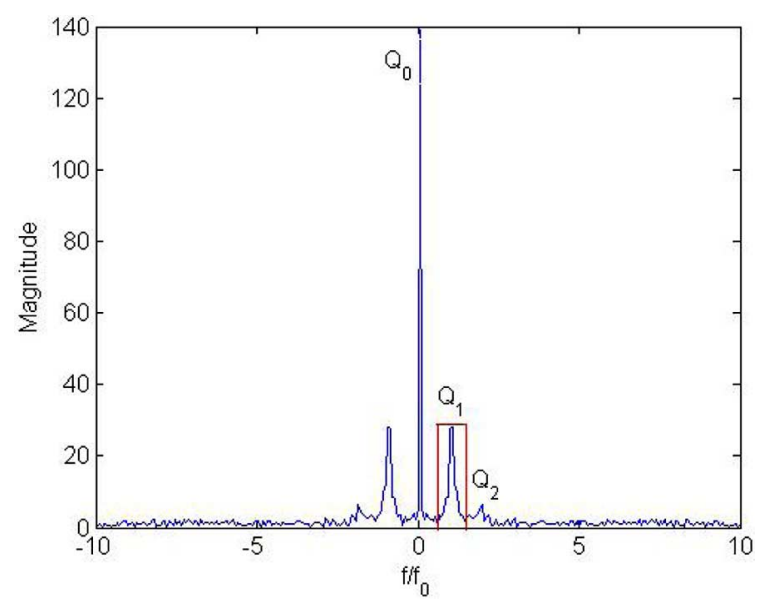

Fig. 2. Magnitude spectrum of the deformed pattern $g(x, y)$.

is the phase modulation including phase introduced by the object. These patterns are known as deformed patterns.

By solving the four equations for the reference patterns, the phase distribution for the reference pattern $\phi_{0}(x, y)$ can be computed as

$$
\phi_{0}(x, y)=\tan ^{-1}\left(\frac{I_{03}(x, y)-I_{01}(x, y)}{I_{00}(x, y)-I_{02}(x, y)}\right) .
$$

The phase distribution for the deformed pattern $\phi(x, y)$ can also be obtained in the similar way. The arctangent operation causes the calculated $\phi_{0}(x, y)$ and $\phi(x, y)$ in a wrapped range between $-\pi$ and $\pi$. It is necessary to recover the unwrapped form by a phase unwrapping operation [22]. The phase distribution related to object under inspection is given by [19]

$$
\Delta \phi(x, y)=\phi(x, y)-\phi_{0}(x, y)=2 \pi f_{0} \overline{C D} .
$$

The height (in millimeters) $h(x, y)$ of the object at a point $(x, y)$ is related to the object phase geometrically. With the use of similar triangles $\Delta E_{p} H E_{c}$ and $\Delta C H D, h(x, y)$ is given by [19]

$$
h(x, y)=\frac{\ell \Delta \phi(x, y)}{\Delta \phi(x, y)-2 \pi f_{0} d} .
$$

If the fringe period of the projected pattern is a constant, (5) can be simplified as [22]

$$
h(x, y)=k \Delta \phi(x, y)
$$

where $k$ is the phase-to-height conversion factor.

\section{B. Principle of Fourier Transform Profilometry}

In Fourier transform profilometry (FTP), only one sinusoidal pattern is required. The captured reference sinusoidal pattern can be expressed as a Fourier series expansion [17]

$$
g_{0}(x, y)=\sum_{n=-\infty}^{\infty} A_{0 n} \exp \left\{i\left[2 \pi n f_{0} y+n \phi_{0}(x, y)\right]\right\}
$$




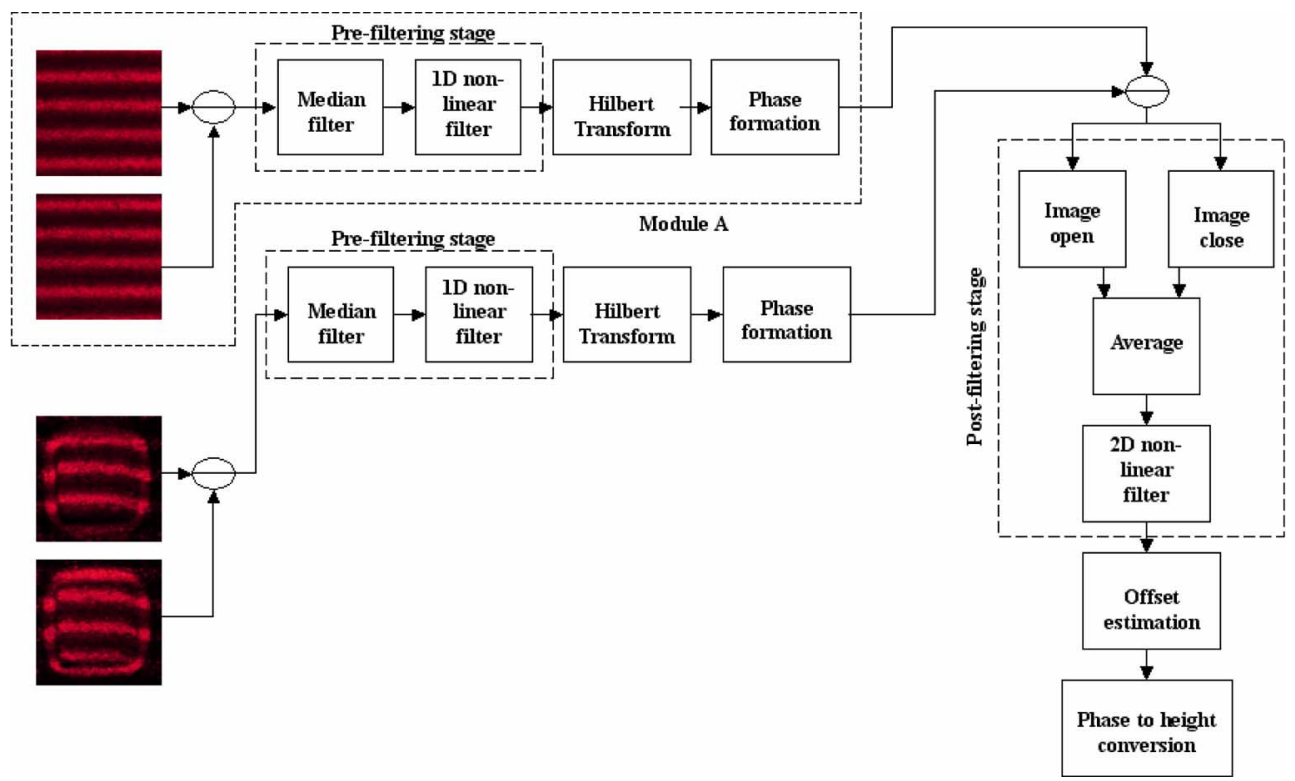

Fig. 3. Block diagram of the two-step phase shift profilometry.

where $A_{0 n}$ are the Fourier series coefficients of the reference pattern. $\phi_{0}(x, y)$ is the phase modulation. If the reflectivity of the referenced plane $r_{0}(x, y)$ is considered, (7) can be expressed as

$$
g_{0}(x, y)=r_{0}(x, y) \sum_{n=-\infty}^{\infty} A_{0 n} \exp \left\{i\left[2 \pi f_{0} y+n \phi_{0}(x, y)\right]\right\}
$$

Similarly, the deformed pattern $g(x, y)$ is given by

$$
g(x, y)=r(x, y) \sum_{n=-\infty}^{\infty} A_{n} \exp \left\{i\left[2 \pi n f_{0} y+n \phi(x, y)\right]\right\}
$$

where $A_{n}$ and $r(x, y)$ are the Fourier series coefficients and the reflectivity for the deformed pattern. $\phi(x, y)$ is the phase modulation. Equation (9) can also be rewritten as

$$
g(x, y)=\sum_{n=-\infty}^{\infty} q_{n}(x, y) \exp \left(2 \pi n f_{0} y i\right)
$$

where $q_{n}(x, y)=A_{n} r(x, y) \exp (n \phi(x, y) i)$.

The object phase $\Delta \phi(x, y)$ carries information about the 3-D object to be measured. It is required to extract it from the patterns. This is achieved by applying 1-D Fourier transform to $g_{0}(x, y)$ and $g(x, y)$ in the y direction. The Fourier transformed $g(x, y)$ is given by [17]

$$
\begin{aligned}
G(f, x) & =\int_{-\infty}^{\infty} g(x, y) \exp (-2 \pi f y i) d y \\
& =\sum_{n=-\infty}^{\infty} Q_{n}\left(f-n f_{0}, x\right) .
\end{aligned}
$$

It is assumed that the height variation of the targeted object is bandlimited by $\pm f_{0}$. Considering the right side of the frequency spectrum in Fig. 2, it is assumed that no undesired signal or noise is contaminated in the spectra at $f_{0}$ i.e., $Q_{1}$. It can be separated from the zeroth spectrum (background term) and higher order harmonics by using bandpass filter. Inverse Fourier transform is then applied to return the images in time domain. The resulted images for the reference and deformed patterns are $\widehat{g}_{0}(x, y)$ and $\widehat{g}(x, y)$, respectively. The equation for $\widehat{g}(x, y)$ is given as

$$
\begin{aligned}
\widehat{g}(x, y) & =q_{1}(x, y) \exp \left(2 \pi f_{0} y i\right) \\
& =A_{1} r(x, y) \exp \left\{\left[2 \pi f_{0} y+\phi(x, y)\right] i\right\} .
\end{aligned}
$$

Multiplication between $\widehat{g}(x, y)$ and the complex conjugate of $\widehat{g}_{0}(x, y)$ is expressed as

$$
\begin{aligned}
& \widehat{g}(x, y) \widehat{g}_{0}^{*}(x, y) \\
& \quad=A_{01}(x, y) A_{1}(x, y) r_{0}(x, y) r(x, y) \exp [\Delta \phi(x, y) i] .
\end{aligned}
$$

The object phase $\Delta \phi(x, y)$ will appear in the imaginary part after applying logarithm to (13). Height-to-phase conversion is carried according to (5) and (6).

\section{Two-Step Phase ShIFT Profilometry}

A novel two-step phase shift profilometry method (2-step PSP) with prefiltering and postfiltering stages is proposed to reconstruct the 3-D profile of solder paste. Fig. 3 shows the outline of the proposed methodology in terms of a block diagram. Two sets of sinusoidal patterns which are $\pi$-out-of-phase are used in the 3-D reconstruction. Two reference patterns which are obtained and processed in an offline manner (module $\mathrm{A}$ in Fig. 3) are stored in the database. Two deformed patterns are processed in an online manner. The imperfectness of the sinusoidal fringe projector can cause the projected fringe patterns not having perfect sinusoidal characteristic. Camera noise can also affect the captured patterns not following the mathematical model closely. Thus, prefiltering and postfiltering stages are included in the proposed profilometry. A defect-free $200 \mu \mathrm{m}$ square solder paste block is used to demonstrate the result after each step. The detail of the system implementation and evaluation will be described in Section IV. 


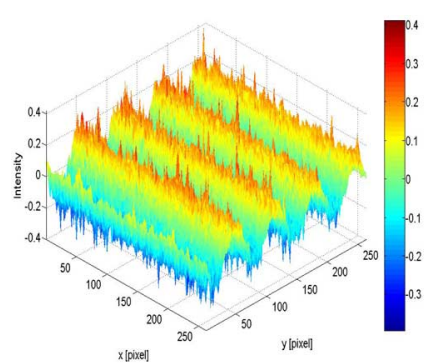

(a) $I_{00 \quad B R}(x, y)$

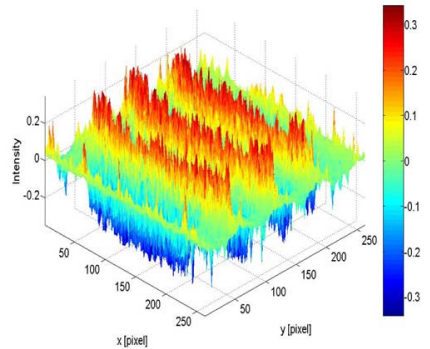

(b) $I_{0} \quad B R(x, y)$
Fig. 4. (a) Background-removed reference pattern and (b) background-removed deformed pattern.

\section{A. Two-Step Phase Shifting Technique}

Two phase-shifted patterns are required in the two-step PSP. The phase increment for each pattern is $\pi$. The equations for the reference and deformed patterns are the same as (1) and (2), respectively, such that $\mathrm{n}=0,2$. The two reference patterns which are obtained and processed in an offline manner are stored in the database, while the two deformed patterns are processed in an online manner.

\section{B. Background Removal}

Ideally, only cosine terms in (1) and (2) are the high-frequency components. $a(x, y), a_{0}(x, y), b(x, y)$, and $b_{0}(x, y)$ are the slow-varying components. For practical situation, the captured fringe patterns contain camera noise. Moreover, the patterns may not follow sinusoidal characteristics very well due to the nonideal sinusoidal pattern projector. As a result, the associated background term is not a simple dc component. This causes overlapping of the fundamental spectrum with the zeroorder spectrum. It is quite difficult to estimate the background term by using just one pattern.

As a result, two fringe patterns which are $\pi$-out-of-phase are utilized to estimate the background term and recover the sinusoidal fringe pattern closer to its ideal form. The deformed pattern $I_{0}(x, y)$ after applying background removal is given by

$$
I_{0 \_B R}(x, y)=\frac{I_{0}(x, y)-I_{2}(x, y)}{2} .
$$

The background removal is applied to $I_{00}(x, y)$ in the same manner to produce $I_{00_{-} B R}(x, y)$. Fig. 4 shows the example of $I_{00_{-} B R}(x, y)$ and $I_{0 \_B R}(x, y)$.

\section{Prefiltering Stage}

The first portion of the prefiltering stage is a 2-D median filter. It is used to remove impulsive noise in the background-removed fringe patterns. Within an $M \times M$ window of pixels of an image (Fig. 5), the values of these pixels are sorted. Let $M$ be an odd number. The central pixel of the window is replaced by the $\left(\left(M^{2}+1\right) / 2\right)^{t h}$ element in the sorted set of neighbors. The window is then applied to other pixel in a left-to-right-top-tobottom approach. At the boundaries, the pixels are symmetrically extended. The results of the reference and deformed patterns after applying 2-D MF are shown in Fig. 6.

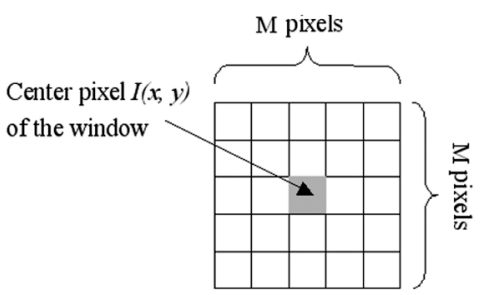

Fig. 5. $N$ by $N$ window used in 2-D median filter.

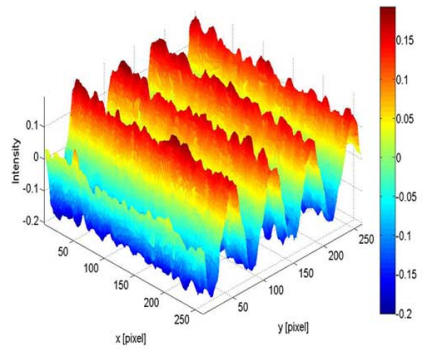

(a) $I_{00 \_B R}(x, y)$ after

applying $2 \mathrm{D}$ median filter

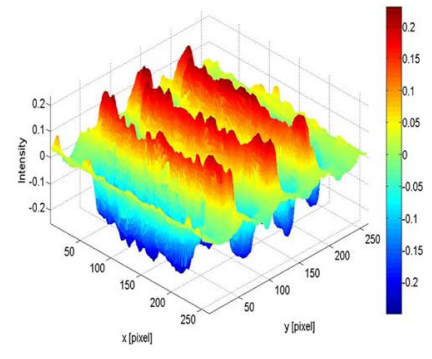

(a) $I_{0_{-} B R}(x, y)$ after

applying $2 \mathrm{D}$ median filter
Fig. 6. (a) Background-removed reference pattern and (b) background-removed deformed pattern after filtering by 2 -D median filter.

Median filter is effective in removing impulsive noise. However, it is not specific in filtering small-amplitude high-frequency noise in the fringe patterns. One of the solutions is to use moving-average filter. Fig. 7 shows the results after applying three different moving-average filters. The window sizes are $1 \times 19,19 \times 1$, and $9 \times 9$. From Fig. 7(b), it can be shown that the edges are blurred after applying the 1-D moving average filter. The sinusoidal signal is blurred in Fig. 7(c) as well. Moreover, the intensity is reduced in Fig. 7(d). From Fig. 7(b), (d), and (f), the small-amplitude noise still exists in the filtered fringe pattern. It is expected that the small-amplitude noise can be reduced in a large extent if a large window is used. However, this will blur the pattern and the reconstructed 3-D profile. Hence, moving average filter is not the best choice to remove the remained small-amplitude noise in the median-filtered fringe pattern.

Some researchers used nonlinear filter to remove small-amplitude high-frequency noise [25], [26]. This filter removes small-amplitude fluctuations while keeping edges sharp. The algorithm is the combination of an averaging filter and a comparator. However, a complex nonlinear filter is required to filter image or signal containing continuous changes (e.g., sinusoidal fringe patterns in our case). Instead of using a 2-D nonlinear filter, a 1-D nonlinear filter is proposed in the second portion of the prefiltering stage such that the major axis of the filter is perpendicular to the direction of the sinusoidal pattern traveling. This 1-D nonlinear filter is named as a 1-D NLFP here. It is also used in the filtering stage in our early work for solder paste inspection using 4-step PSP [27].

The size of the filter is 1 by $2 N+1$. The window of the $1 \mathrm{D}$ nonlinear filter is defined in Fig. 8. A image $I(x, y)$ is scanned in a left-to-right-top-to-bottom manner. The resultant after applying 1-D NLFP to an image $I(x, y)$ is written as [27] 


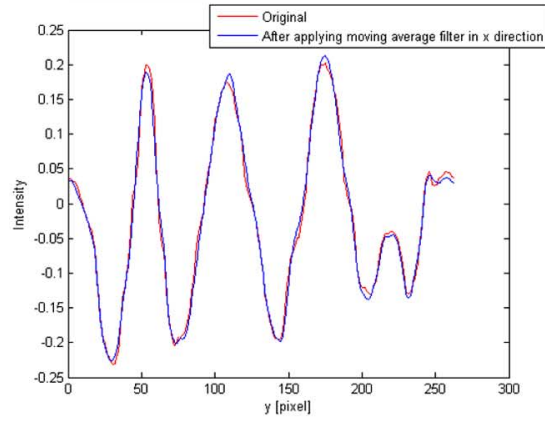

(a)

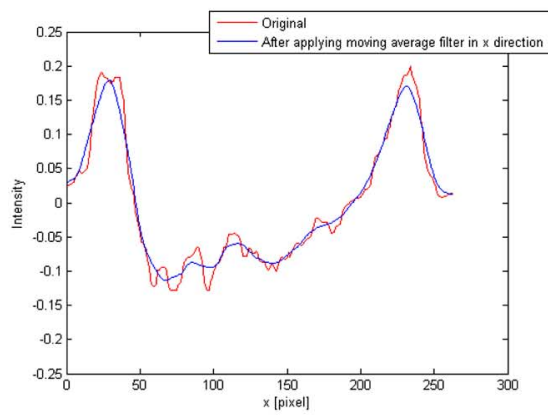

(b)

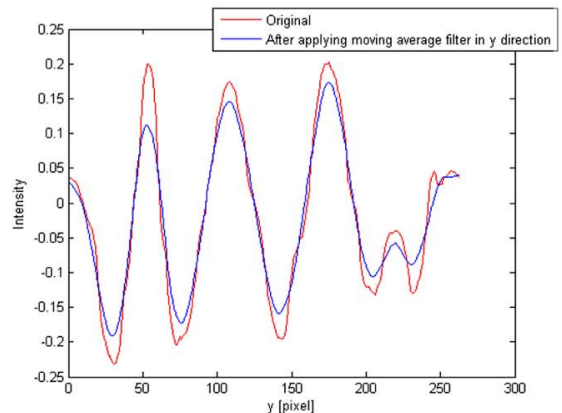

(c)

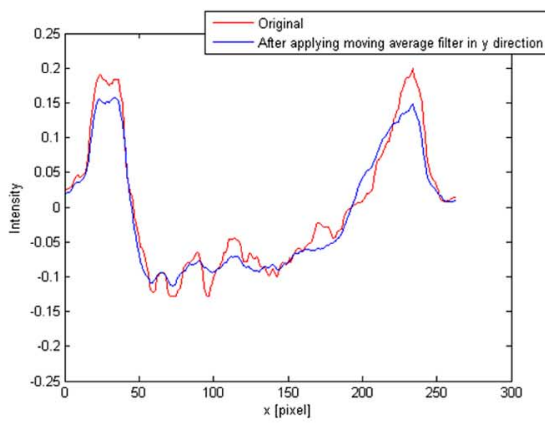

(d)

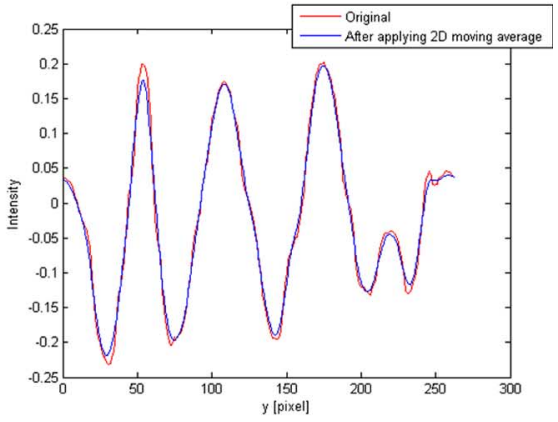

(e)

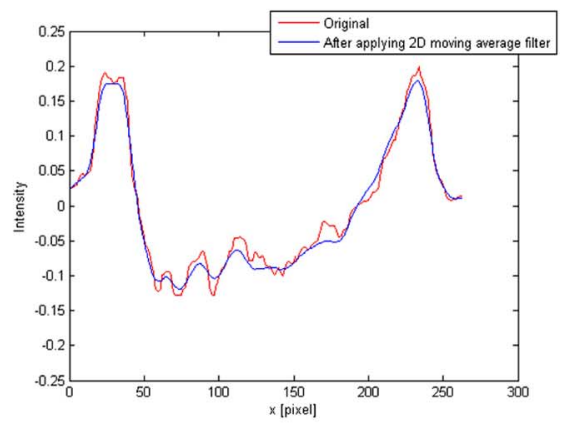

(f)

Fig. 7. Cross-sectional views of the results for applying three different moving average filters for median filtered $g_{0-B R}(x, y)$ at $x=132$ pixels and $y=$ 132 pixels. (a), (b) Using 1-D $1 \times 19$ moving average filter. (c), (d) Using 1-D $19 \times 1$ moving average filter. (e), (f) Using 2-D $9 \times 9$ moving average filter. (a), (b), (c) Results at $x=132$ pixels. (d), (e), (f) Results at $y=132$ pixels.

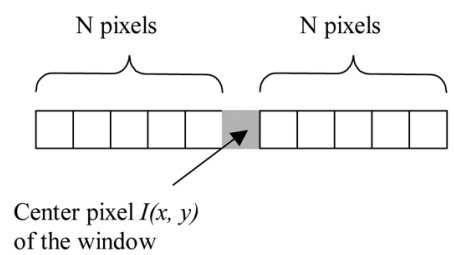

Fig. 8. Window of the 1-D non-linear filter.

$$
I_{\mathrm{NLFP}}(x, y)=I(x, y)-\text { Noise }(x, y)
$$

such that

$$
\text { Noise }(x, y)=\frac{\sum_{k=-N+1}^{N-1} F(I(x, y)-I(x, y+k))}{2 N}
$$

and

$$
F(u)= \begin{cases}u, & \text { if } u \leq \text { Threshold } \\ 0, & \text { otherwise }\end{cases}
$$

where $N$ is an odd number and function $F(u)$ is a threshold filter.

Fig. 9 shows the results after applying conventional 2-D nonlinear filter with $N=9$ and 2-D nonlinear filter with $N=19$ and also the proposed 1-D NLFP with $N=19$. It can be shown that the conventional 2-D non-linear filter cannot remove the small-amplitude-high-frequency noise. The proposed 1-D NLFP filter can remove small-amplitude fluctuation effectively while preserving sharp edges. Fig. 10 shows the reference and deformed patterns after passing through the prefiltering stage.

\section{In-Quadrature Component}

The background-removed fringe pattern has been properly filtered by the prefiltering stage so as to remove small-amplitude-high-frequency fluctuation causing high-order harmonics. The fringe pattern is then prepared for object phase reconstruction. There is no need to utilize bandpass filter to extract fundamental spectrum as FTP. The in-quadrature component of the processed fringe pattern can be used to obtain the embedded 3-D information. It can be obtained by applying 1-D Hilbert transform (HT) to the processed fringe pattern. It is assumed that the height variation of the targeted object is bandlimited by $\pm f_{0}$. Considering the deformed pattern after passing through prefiltering stage as $\tilde{I}_{0}(x, y)$, its in-quadrature component $\tilde{I}_{1}(x, y)$ is obtained from Hilbert transform as [20]

$$
\tilde{I}_{1}(x, y)=-H T\left\{\hat{I}_{0}(x, y)\right\}=-\frac{1}{\pi t} * \hat{I}_{0}(x, y) .
$$

Fig. 11 shows the estimated in-quadrature components of the reference and deformed patterns.

\section{E. Object Phase Reconstruction}

The deformed phase $\phi(x, y)$ can be obtained from the following equation:

$$
\phi(x, y)=\tan ^{-1}\left\{\frac{\tilde{I}_{1}(x, y)}{\widehat{I}_{0}(x, y)}\right\} .
$$




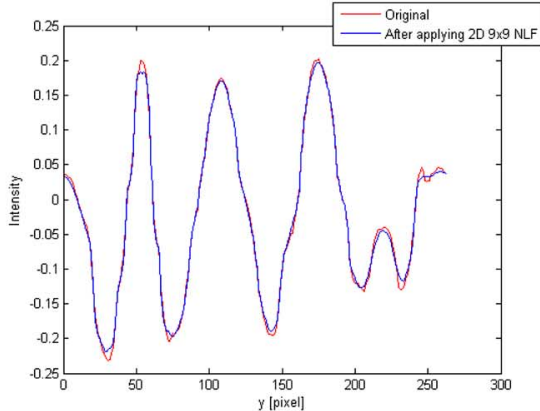

(a)

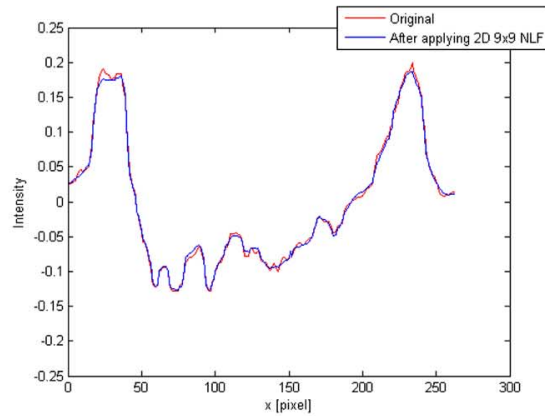

(b)

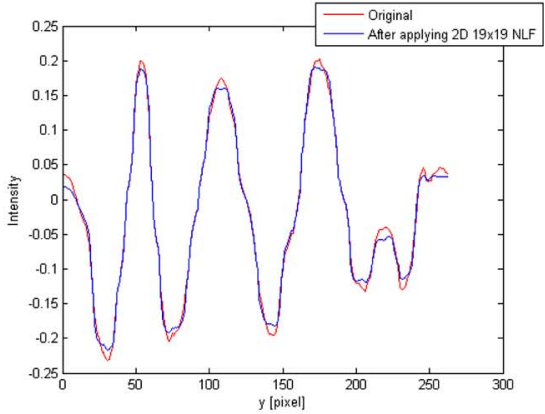

(c)

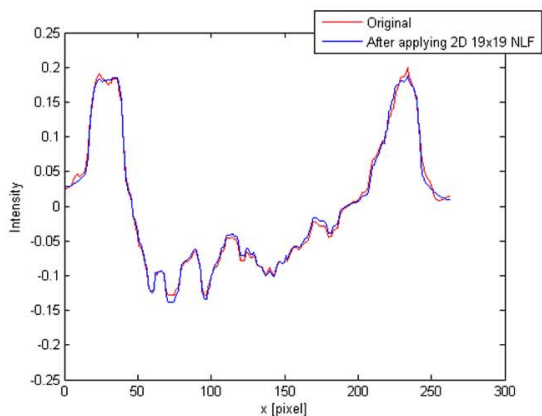

(d)

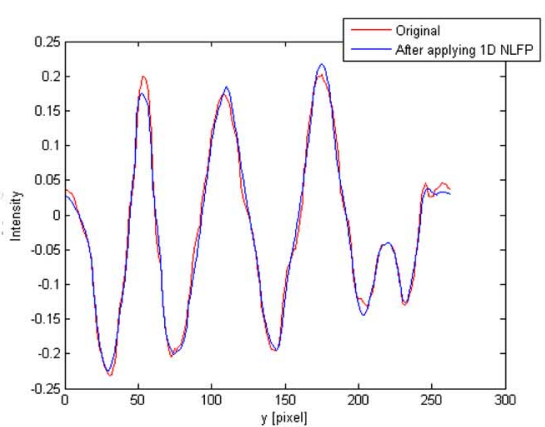

(e)

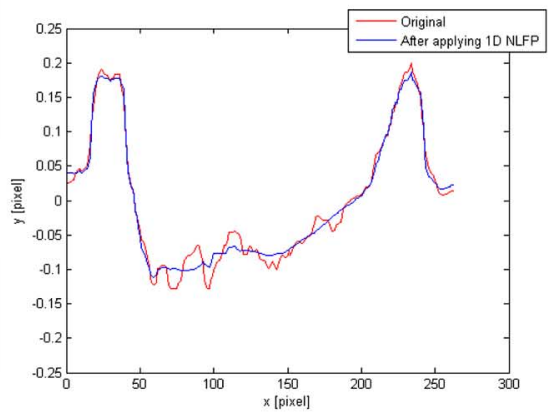

(f)

Fig. 9. Cross-sectional views of the results for applying three different nonlinear filters for median filtered $g_{0} B R(x, y)$ at $x=132$ pixels and $y=132$ pixels. (a), (b) Using 2-D nonlinear filter with $N=9$. (c), (d) Using 2-D nonlinear filter with $N=19$. (e), (f) Using 1-D NLFP with $N=19$. (a), (b), (c) Results at $x=132$ pixels. (d), (e), (f) Results at $y=132$ pixels.

The reference phase $\phi_{0}(x, y)$ is obtained in the similar manner. Phase unwrapping [22] is also required to apply for (19). The reconstructed object phase is shown in Fig. 12.

\section{F. Postfiltering}

Due to the nonideal sinusoidal characteristic of the projected pattern, the reconstructed profile of the object may contain undesired features. From Fig. 12, the reconstructed 3-D profile contains fringe-like structures. A simple moving average filter cannot be used to remove the artifacts effectively as this will blur the reconstructed 3-D profile. The fringe-like artifacts is not a kind of high-frequency noise such that nonlinear filter is no longer effective in removing the artifacts. An average of grayscale image open and image close is proposed to remove the fringe-like structures. The filtered object phase $\Delta \phi(x, y)$ is given by

$$
\Delta \phi_{\text {filtered }}(x, y)=\frac{\Delta \phi(x, y) \circ h(x, y)+\Delta \phi(x, y) \bullet h(x, y)}{2}
$$

where $\Delta \phi(x, y) \circ h(x, y)$ and $\Delta \phi(x, y) \bullet h(x, y)$ are the image open and image close of $\Delta \phi(x, y)$ by the structural element $h(x, y)$. It is then smoothed by a 2-D nonlinear filter. The result of the object phase after applying image open and image close are shown in Fig. 13(a) and (b). The overall result after applying postfiltering is shown in Fig. 13(c).

\section{G. Offset Estimation}

The object phase $\Delta \phi(x, y)$ is the difference between deformed phase $\phi(x, y)$ and reference phase $\phi_{0}(x, y)$. As a result, the volume of solder paste cannot be measured relative to the $\mathrm{PCB}$. One way to solve this problem is to segment the recon- structed solder paste from the background plane by estimating the offset value between the reference plane and PCB. The offset compensated object phase $\Delta \phi_{o c}(x, y)$ is given by

$$
\Delta \phi_{o c}(x, y)=(\Delta \phi(x, y)-\text { Offset }) M(x, y)
$$

where a solder paste mask $M(x, y)$ is the projection of the 3-D solder paste profile onto the $x$ - $y$ plane. The solder paste mask is obtained by thresholding the top view of the solder paste block such that it is imaged from a well-illuminated scene without projecting fringe pattern. Considering the size of the imaged patterns is $m \times n$, the average phase value of the non-solder region of the reconstructed object can be used as an estimation of the offset value. The offset value is calculated as

$$
\text { Offset }=\frac{\sum_{x=0, y=0}^{m-1, n-1} \Delta \phi(x, y)(1-M(x, y))}{\text { Number of white pixels in }(1-M(x, y))} \text {. }
$$

Fig. 14 shows the reconstructed 3-D solder paste profile with offset estimation.

\section{H. Object Phase to Height Conversion}

The reconstructed 3-D profiles of solder paste blocks are converted from the offset compensated object phase $\Delta \phi_{o c}(x, y)$ to height $h(x, y)$ using (5) or (6). In Section IV, it will be shown that the fringe period can be considered as a constant, so (6) will be used in the following section.

\section{SYSTEM IMPLEMENTATION AND EVALUATIONS}

The experimental setup for the two-step phase shift profilometry is shown in Fig. 15. The optical system is simple, compact, 


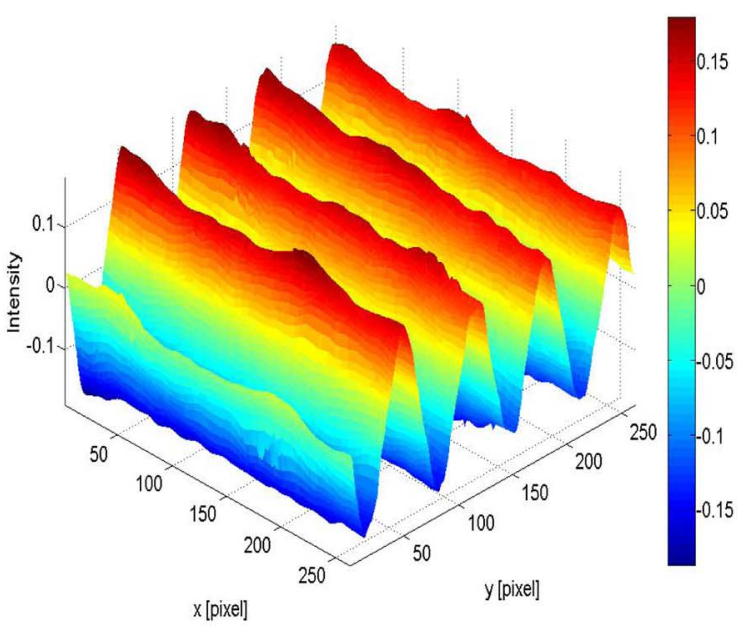

(a) $I_{00 \_B R}(x, y)$ after passing through prefiltering stage

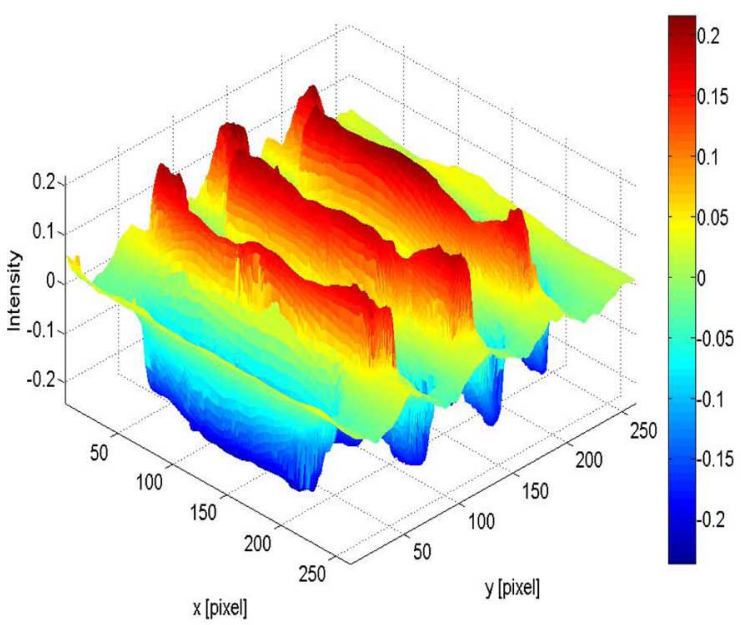

(b) $I_{0_{B} B R}(x, y)$ after passing through prefiltering stage

Fig. 10. Background-removed reference and deformed patterns after applying 2-D median filter followed by 1-D NLFP (prefiltering stage).

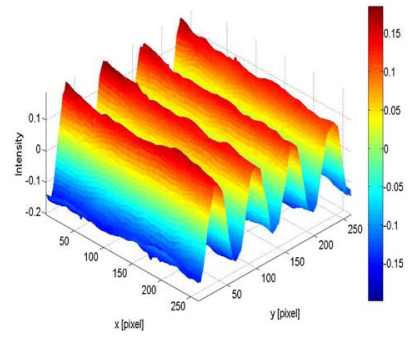

(a)

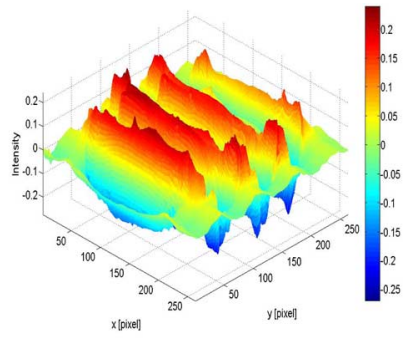

(b)
Fig. 11. (a) Estimated in-quadrature components of the processed reference pattern and (b) deformed pattern.

and relatively low-cost. A multiple-line laser projector with 635-nm wavelength (A) is used in the profilometry. The multiple-line pattern is defocused through the use of an external lens (B) so that sinusoidal fringe pattern can be generated. A dimmable white light emitting diode (LED) light (C) is used to provide a well-illuminated environment to capture top view of solder paste samples (D). A $1280 \times 960$ CCD camera with an IEEE 1394 interface (E) is used as the imaging device.

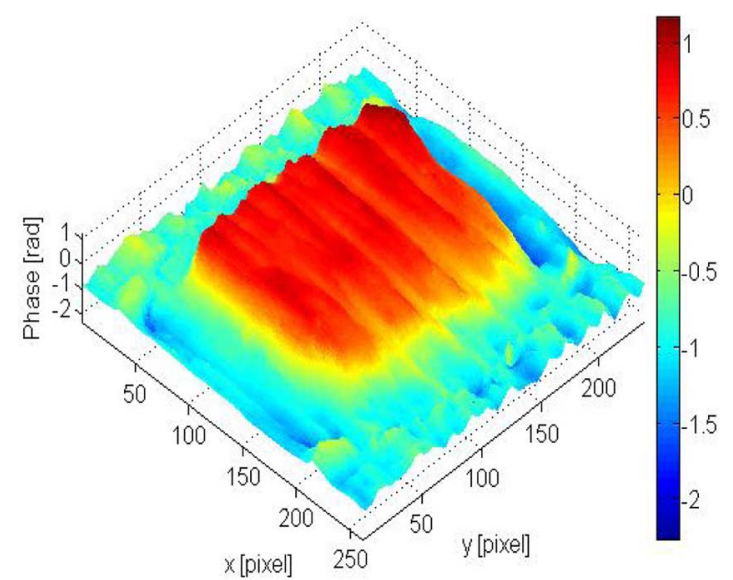

Fig. 12. Reconstructed object phase.

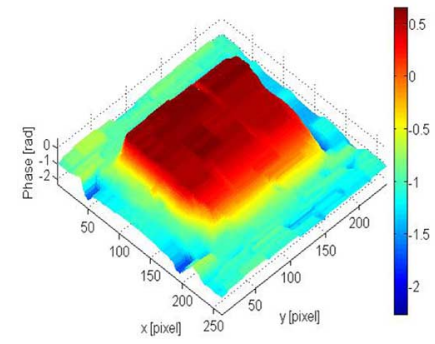

(a)

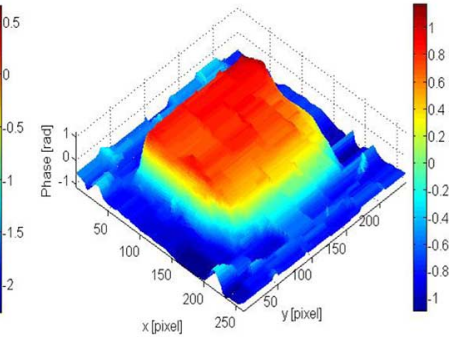

(b)

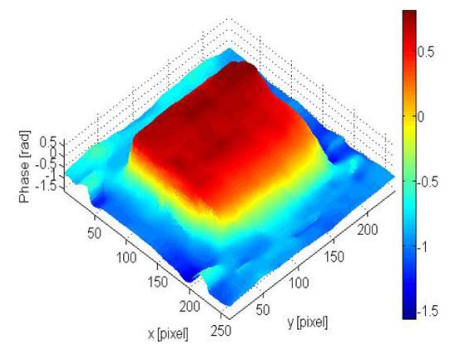

(c)

Fig. 13. (a) Reconstructed object phase after applying image open, (b) image close, and (c) after passing through a postfiltering stage.

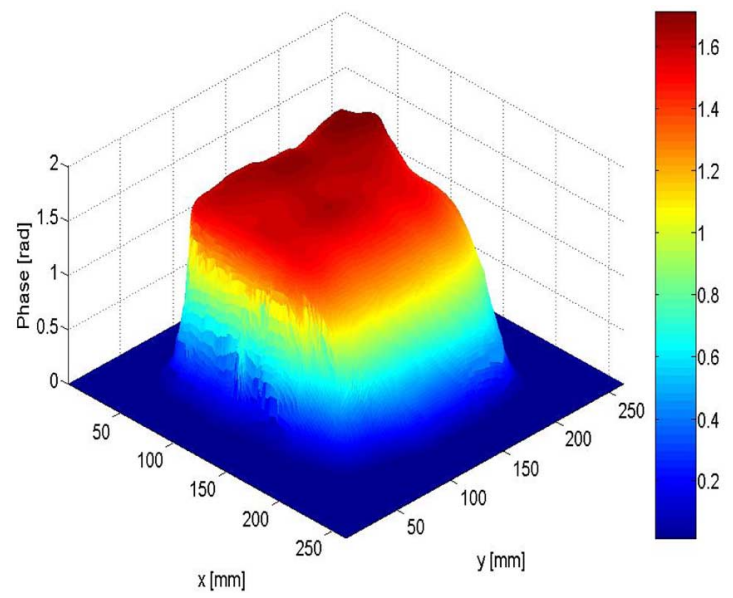

Fig. 14. Reconstructed object phase with offset estimation.

The magnification ratio of the imaging device is 0.0078 , i.e., the width of each square pixel is $7.8 \mu \mathrm{m}$. The phase shifting 


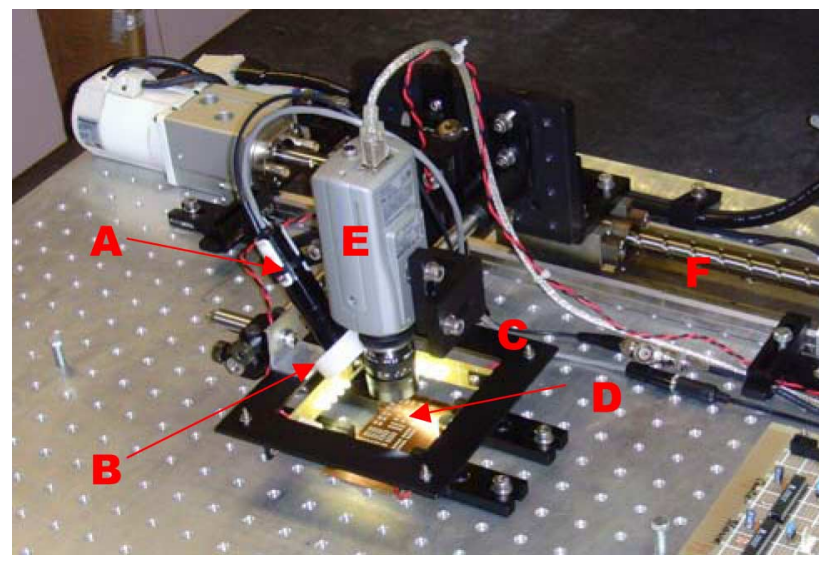

Fig. 15. Experimental setup for the two-step phase shift profilometry.

mechanism is achieved by mounting the laser projector on a linear servo track (F) with 1- $\mu \mathrm{m}$ resolution. The fringe period is 64 pixels, i.e., $0.5 \mathrm{~mm}$. The size of the imaged fringe pattern is $263 \times 263$ pixels, i.e., $2.05 \mathrm{~mm} \times 2.05 \mathrm{~mm}$.

The fringe period can be measured by using a piece of white reference plane marked with a black straight reference line. The white LED light is switched on so as to provide a well-illuminated environment to inspect the reference black line. The procedure is as follows.

1) Placing the reference plane under the imaging device with projector on. The plane is aligned such that the black line settles between two peaks near the upper boundary of the imaging region. This line is named as CalRef1_1.

2) The plane is moved to another position so that the black reference line settles between the next trough near the upper boundary of the imaging region. This line is named as CalRef1_2.

3) The same procedure is carried out such that two more lines CalRef2_1 and CalRef2_2 are obtained near the lower boundary of the imaging region.

By thresholding the captured images, the reference lines can be extracted. In order to have single-pixel-width reference lines, the central line of the black reference line can be determined by the following weighted averaging technique [8]:

$$
\begin{aligned}
& \text { Center of the black reference line } \\
& =\frac{\sum(\text { Pixel Column Position x Pixel Intensity })}{\sum(\text { Pixel Intensity })} .
\end{aligned}
$$

Fig. 16 shows the distribution of the four lines. The average top fringe period and the average bottom fringe period equal to 62.79 pixels and 64.99 pixels, respectively. The difference between average top fringe period and average bottom fringe period is 2.2 pixels. Thus, the fringe period can be considered to be a constant number which equals to 64 pixels.

As a comparison, the same $200-\mu \mathrm{m}$ square-type solder paste block which is used in Section III is also reconstructed using four-step PSP and FTP. The reconstructed object phase distributions are shown in Figs. 17 and 18, respectively. From Fig. 17, the outline of the reconstructed object phase using four-step

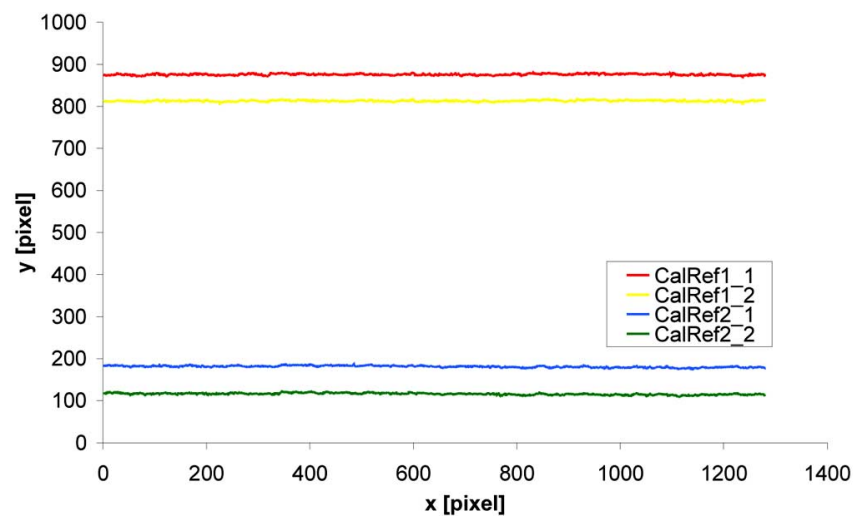

Fig. 16. Distribution of the four reference lines.

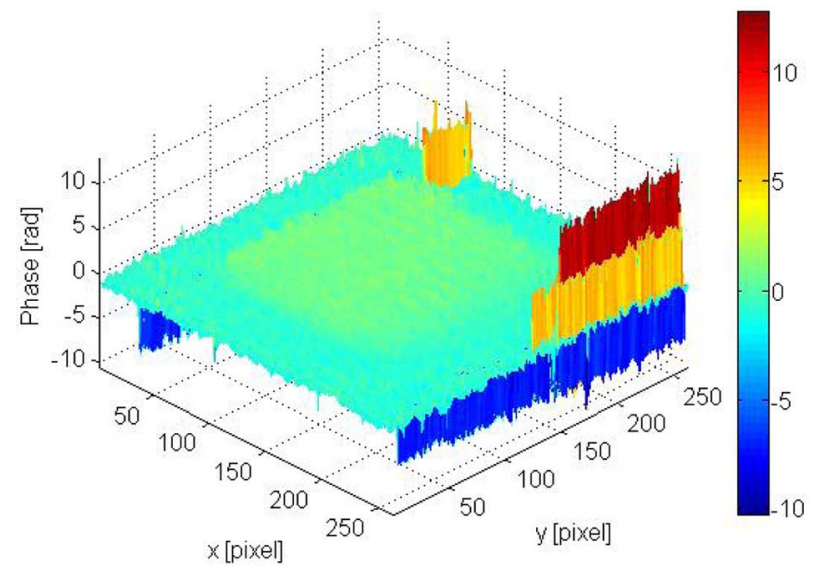

Fig. 17. Reconstructed object phase using four-step PSP.

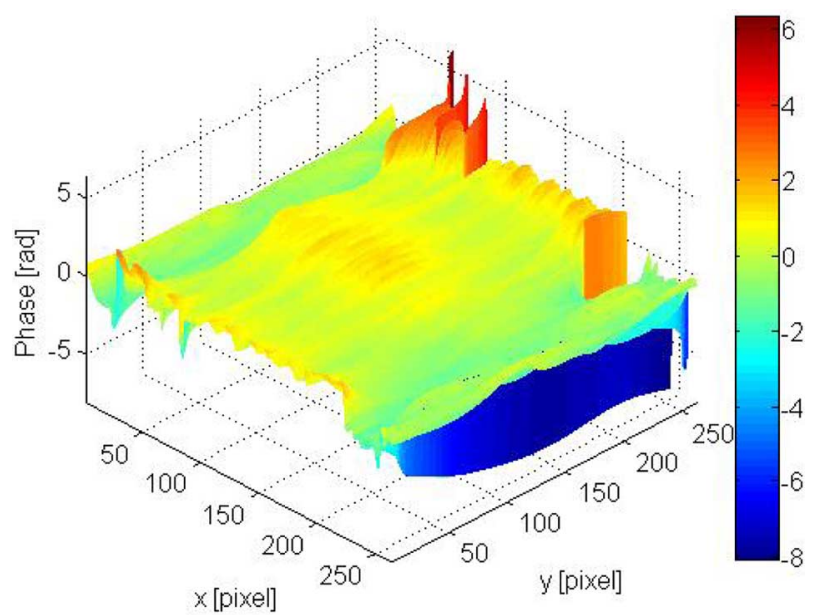

Fig. 18. Reconstructed object phase using FTP.

PSP can be observed. However, there are phase unwrapping errors. This is due to the fact that noise in the captured patterns can easily trigger the phase unwrapping procedure. For FTP, phase unwrapping errors also exist in the reconstructed object phase distribution. The outline of the object phase distributions is hardly observed. FTP has an important assumption about the spectrum of the imaged fringe pattern. It is assumed that no undesired signal or noise is contaminated in the spectrum at $f_{0}$. 

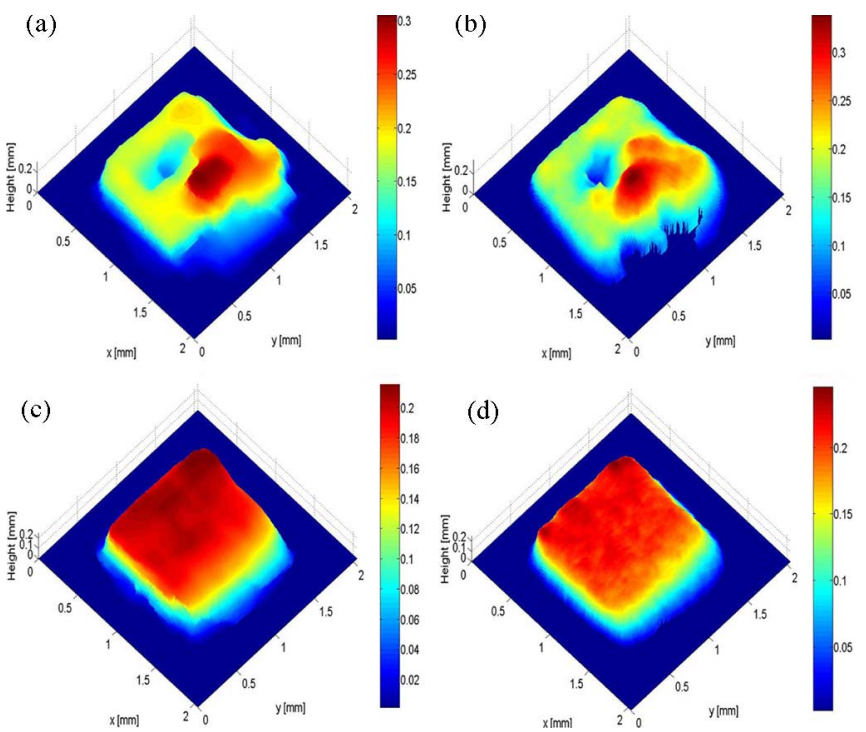

Fig. 19. Comparisons of the 3-D profiles between $(\mathrm{a}, \mathrm{c})$ reconstructed solder paste blocks and $(b, d)$ reference solder paste blocks using $200-\mu \mathrm{m}$ stencil $[(\mathrm{b})$ and (d) are the defective and defect-free samples, respectively].

It can be concluded that undesired signal or noise has contaminated in the spectrum at $f_{0}$. The source of contamination can be due to noisy imaged fringe patterns and nonideal sinusoidal characteristics of the projected pattern.

In order to evaluate the performance of the proposed profilometry, a point-type laser scanner is used to measure the 3-D profiles of several solder paste blocks. These profiles are used as the reference 3-D profiles for the evaluation. The scanner consists of a laser displacement sensor which is mounted on an $x-y$ table. The $z$-resolution of the laser sensor and the resolution of the $x-y$ table is $0.05 \mu \mathrm{m}$ and $10 \mu \mathrm{m}$, respectively. The $x y$-resolution of the reconstructed 3-D solder paste profile can be reduced to the same as that of the laser scanner by nearest neighbor decimation [24]. Three groups of five defect-free and three defective solder paste blocks are used in the evaluation. The solder paste samples are square-type solder paste blocks with three stencil thicknesses, namely $150 \mu \mathrm{m}, 200 \mu \mathrm{m}$, and $250 \mu \mathrm{m}$.

The 3-D profiles of the reconstructed solder paste blocks $h(x, y)$ are evaluated using volume accuracy and RMSE against the corresponding reference 3 -D profiles $h_{r e f}(x, y)$ which are obtained from the laser scanner. Volume and RSME [24] of each reconstructed solder paste block are defined as

$$
\text { Volume }=w^{2} \sum_{x=0}^{m-1} \sum_{y=0}^{n-1} h(x, y)
$$

and

$$
\mathrm{RMSE}=\sqrt{\frac{\sum_{x=0}^{m-1} \sum_{y=0}^{n-1}\left(h_{\mathrm{ref}}(x, y)-h(x, y)\right)^{2}}{m n}}
$$

respectively, where $w$ are the width of each pixel in millimeters. The size of the imaged fringe patterns is $m \times n$. The corresponding reference solder paste block volume is also calculated using (24) such that $h(x, y)$ and $w$ become $h_{r e f}(x, y)$ and the resolution of the $x-y$ table, respectively.
TABLE I

MeAsurement ACCuRaCy OF THE PROPOSEd Two-STEP PSP USING (a) $150-\mu \mathrm{m}$ SOLDER PASTE BLOCKS, (b) 200- $\mu \mathrm{m}$ SOLDER PASTE BLOCKS, AND (c) $250-\mu \mathrm{m}$ SOLDER PASTE BLOCKS

\begin{tabular}{|c|c|c|c|c|}
\hline \multirow{5}{*}{ Normal } & $\begin{array}{c}\text { Reference } \\
\text { volume } \\
\left(\mathrm{mm}^{3}\right)\end{array}$ & $\begin{array}{c}\text { Measured } \\
\text { volume } \\
\left(\mathrm{mm}^{3}\right)\end{array}$ & $\begin{array}{c}\text { Volume } \\
\text { accuracy } \\
(\%)\end{array}$ & $\begin{array}{c}\text { RMSE } \\
(\mathrm{um})\end{array}$ \\
\hline \multirow{5}{*}{ Defective } & 0.2141 & 0.2068 & 96.47 & 15.4 \\
\cline { 2 - 5 } & 0.2155 & 0.2116 & 98.19 & 19 \\
\cline { 2 - 5 } & 0.2079 & 0.2064 & 99.28 & 15.5 \\
\cline { 2 - 5 } & 0.1648 & 0.1743 & 94.35 & 17.8 \\
\cline { 2 - 5 } & 0.2240 & 0.2233 & 99.69 & 21.1 \\
\cline { 2 - 5 } & 0.2082 & 0.1851 & 88.90 & 25 \\
\cline { 2 - 5 } & 0.2741 & 0.2591 & 94.52 & 16.3 \\
\hline & 0.1990 & 0.1641 & 89.62 & 25.9 \\
\hline
\end{tabular}

(a)

\begin{tabular}{|c|c|c|c|c|}
\hline \multirow{5}{*}{ Normal } & $\begin{array}{c}\text { Reference } \\
\text { volume } \\
\left(\mathrm{mm}^{3}\right)\end{array}$ & $\begin{array}{c}\text { Measured } \\
\text { volume } \\
\left(\mathrm{mm}^{3}\right)\end{array}$ & $\begin{array}{c}\text { Volume } \\
\text { accuracy } \\
(\%)\end{array}$ & $\begin{array}{c}\text { RMSE } \\
(\mathrm{um})\end{array}$ \\
\hline \multirow{5}{*}{ Defective } & 0.2968 & 0.2843 & 95.79 & 15.2 \\
\cline { 2 - 5 } & 0.3574 & 0.3454 & 96.64 & 31.6 \\
\cline { 2 - 5 } & 0.3155 & 0.2872 & 91.03 & 23.8 \\
\cline { 2 - 5 } & 0.3318 & 0.3014 & 90.84 & 25.1 \\
\cline { 2 - 5 } & 0.3098 & 0.2966 & 95.74 & 19.7 \\
\cline { 2 - 5 } & 0.3025 & 0.2958 & 97.79 & 22.9 \\
\cline { 2 - 5 } & 0.3717 & 0.3371 & 90.69 & 35.2 \\
\hline & 0.2825 & 0.3193 & 97.20 & 29.4 \\
\hline
\end{tabular}

(b)

\begin{tabular}{|c|c|c|c|c|}
\hline \multirow{5}{*}{ Normal } & $\begin{array}{c}\text { Reference } \\
\text { volume } \\
\left(\mathrm{mm}^{3}\right)\end{array}$ & $\begin{array}{c}\text { Measured } \\
\text { volume } \\
\left(\mathrm{mm}^{3}\right)\end{array}$ & $\begin{array}{c}\text { Volume } \\
\text { accuracy } \\
(\%)\end{array}$ & $\begin{array}{c}\text { RMSE } \\
(\mathrm{um})\end{array}$ \\
\hline \multirow{5}{*}{ Defective } & 0.3744 & 0.3915 & 95.43 & 35.2 \\
\cline { 2 - 5 } & 0.3913 & 0.3883 & 99.23 & 22.2 \\
\cline { 2 - 5 } & 0.3847 & 0.3565 & 92.67 & 23.9 \\
\cline { 2 - 5 } & 0.4199 & 0.4029 & 99.76 & 28.7 \\
\cline { 2 - 5 } & 0.3876 & 0.3553 & 91.17 & 34.2 \\
\cline { 2 - 5 } & 0.3753 & 0.3667 & 97.77 & 28.3 \\
\cline { 2 - 5 } & 0.3308 & 0.3121 & 94.43 & 35.9 \\
\hline & 0.3472 & 0.3127 & 95.51 & 33.3 \\
\hline
\end{tabular}

(c)

Fig. 19 shows two examples of reconstructed $200-\mu \mathrm{m}$ solder paste blocks and their reference solders paste blocks. The volume accuracy and the RMSE of the reconstructed solder paste blocks for $150 \mu \mathrm{m}, 200 \mu \mathrm{m}$, and $250 \mu \mathrm{m}$ are shown in Table I(a)-(c), respectively.

\section{CONCLUSION}

A new two-step phase shift profilometry (2-step PSP) with filtering stages is proposed to reconstruct 3-D profile of solder paste. This method has the following advantages:

1) It requires two less fringe patterns when compared with the 4-step PSP. This is an advantage in practical implementation, as the time required for image acquisition would be halved.

2) In the current setup, the optical system is simple and compact. A moving mechanism is used to acquire a second 
image using the laser-line projector for generating an $\pi$ out-of-phase fringe pattern. However, the requirement can be eliminated by the use of a second laser-line projector. In other words, the two $\pi$ out-of-phase patterns can be generated using two laser-line projectors. This will be considered in our future research.

3) Comparing to the FTP, the 2-step PSP has better performance in reconstructing 3-D profile.

4) There is no need to use reference solder paste samples like the inspection method based on fuzzy logic and artificial neural network.

Due to the nonideal sinusoidal characteristic of the laser projector, the associated background term is not a simple dc component. This causes overlapping of the fundamental spectrum with the zero order spectrum. The background term is removed by taking the average of the 0 phase-shifted and $\pi$ phase-shifted fringe patterns. As the imaged fringe patterns are properly filtered by the prefiltering stage, the overlapping of the fundamental spectrum with its neighbor is reduced. There is no need to use a bandpass filter to extract the fundamental spectrum. Hilbert transform is used to obtain in-quadrate component of the filtered fringe pattern. A postfiltering stage is then used to reconstruct an appropriate 3-D surface profile. The performance of the 2-step PSP profilometry was evaluated using both defect-free and defective solder paste blocks. Three stencil print thickness were used, namely $150 \mu \mathrm{m}, 200 \mu \mathrm{m}$, and $250 \mu \mathrm{m}$. The percentages of the average volume accuracy for the $150-\mu \mathrm{m}, 200-\mu \mathrm{m}$, and $250-\mu \mathrm{m}$ defect-free and defective solder paste blocks are $96.15 \%, 94.64 \%, 96.01 \%, 94.37 \%, 95.39 \%$, and $96.53 \%$, respectively.

\section{REFERENCES}

[1] H. K. Brown, L. A. Martin-Vega, W. H. Shaw, J. G. Madry, and C. L. Taylor, "Utilization of sensory feedback during SMT assembly," in Proc. IEEE Southcon, 1994, pp. 349-352.

[2] R. Kelley and D. Tan, "A 3-D solder paste inspection strategy for CSPs and 0201s," in APEX 2001 Solder Printing Workshop Handout.

[3] J. Pan, G. L. Tonkay, R. H. Storer, R. M. Sallade, and D. J. Leandri, "Critical variables of solder paste stencil printing for micro-BGA and fine-pitch QFP," in Proc. Electron. Manuf. Technol. Symp., 1999, pp. 94-101.

[4] J. Pan, G. L. Tonkay, R. H. Storer, R. M. Sallade, and D. J. Leandri, "Critical variables of solder paste stencil printing for micro-BGA and fine pitch QFP," IEEE Trans. Electron. Packag. Manuf., vol. 27, no. 2, pp. 125-132, Apr. 2004.

[5] S. Venkateswaran and K. Srihari, "A realtime process control system for solder paste stencil printing," in Proc. IEEE/CPMT Int. Electron. Manuf. Technol. Symp., 1997, pp. 62-67.

[6] "2D v. 3D, That is the Question," PCB Pulse Article [Online]. Available: http://www.home.agilent.com.

[7] R. R. Lathrop, Jr, "Solder paste print qualification using laser triangulation," IEEE Trans. Compon., Packag. Manuf. Technol., vol. 20, no. 3, pp. 174-182, Jun. 1997.

[8] J. P. Lavelle, S. R. Schuet, and D. J. Schuet, "High speed 3D scanner with real-time 3D processing," in Proc. ISA/IEEE Sens. for Industry Conf., 2004, pp. 102-108.
[9] T. E. Kim and J. Choi, "3-D shape recovery of hybrid reflectance surface using indirect diffuse illumination," in IEEE Proc. Int. Conf. Image Process., 1995, vol. 2, pp. 354-357.

[10] R. Zhang, P. S. Tsai, J. E. Cryer, and M. Shah, "Shape from shading: A survey," IEEE Trans. Pattern Anal. Mach. Intell., vol. 21, no. 8, pp. 690-706, Aug. 1999.

[11] K. W. Ko, Y. J. Roh, and H. S. Cho, "A neural network approach to the inspection of ball grid array solder joints on printed circuit boards," in Proc. IEEE-INNS-ENNS Int. Joint Conf. Neural Networks, 2000, pp. 233-238

[12] F. C. Yang, C. H. Kuo, J. J. Wing, and C. K. Yang, "Reconstruction the 3D solder paste surface model using image processing and artificial neural network," in Proc. IEEE Int. Conf. Syst., Man, Cybern., 2004, vol. 3, pp. 3051-3056.

[13] G. Acciana, G. Brunetti, and G. Fornarelli, "A multiple neural network system to classify solder joints on integrated circuits," Int. J. Comput. Intell. Res., vol. 2, pp. 337-348, 2006.

[14] K. W. Ko, H. S. Cho, J. H. Kim, and J. S. Kim, "Solder joints inspection using neural network and fuzzy rule-based classification," in Proc. IEEE/RSJ Int. Conf. Intell. Robotics, Syst., 1998, vol. 3, pp. 1565-1570.

[15] K. W. Lo and H. S. Cho, "Solder joints inspection using a neural network and fuzzy rule-based classification method," IEEE Trans. Electron. Packag. Manuf., vol. 23, pp. 93-103, 2000.

[16] K. M. Jeong, J. S. Kim, K. C. Koh, and H. S. Cho, "Development of PMP system for high speed measurement of solder paste volume on printed circuit boards," Proc. SPIE, vol. 4564, pp. 250-259, 2001.

[17] M. Takeda and K. Mutoh, "Fourier transform profilometry for the automatic measurement of 3-D object shapes," Appl. Opt., vol. 22, no. 24, pp. 3977-3982, 1983.

[18] L. D. Stefano and F. Boland, "Three-dimensional inspection of printed circuit boards using phase profilometry," in Proc. EUSIPCO, 1996, pp. $1805-1808$.

[19] L. D. Stefano, "Solder paste inspection by structured light methods based on phase measurement," Proc. SPIE, vol. 2899, pp. 702-713, 1996.

[20] D. A. Zweig and R. E. Hufnagel, "A Hilbert transform algorithm for fringe-pattern analysis," Proc. SPIE Adv. Opt. Manuf. Testing, pp. 295-302, 1990

[21] H. N. Yen and D. M. Tsai, "A fast full-filed 3D measurement system for BGA coplanarity inspection," Int. J. Adv. Manuf. Technol., pp. 132-139, 2004.

[22] H.-N. Yen, D.-M. Tasi, and J.-Y. Yang, "Full-filed 3-D measurement of solder pastes using LCD-based phase shifting techniques," IEEE Trans. Electron. Packag. Manuf., vol. 29, no. 1, pp. 50-57, Dec. 2006.

[23] X. Wu, W. K. Chung, H. Tong, J. Cheng, and Y. S. Xu, "A new solder paste inspection device: Design and algorithm," in IEEE Int. Conf. Robot. Autom., 2007, pp. 680-685.

[24] R. C. Gonzalez and R. E. Woods, Digital Image Processing, 2nd ed. Upper Saddle River, NJ: Prentice-Hall, 2002.

[25] H. Watabe, Y. Arakawa, and K. Arakawa, "Nonlinear filters for multimedia applications," in Proc. IEEE Int. Conf. Image Process., 1999, vol. 3, pp. 174-178.

[26] K. Arakawa, "Nonlinear digital filters for beautifying facial images in multi-media systems," in IEEE Proc. Int. Symp. Circuits Syst., 2004, vol. 5, pp. 429-432.

[27] T. W. Hui and G. K. H. Pang, "3D profile reconstruction of solder paste based on phase shift profilometry," in 5th IEEE Int. Conf. Ind. Informatics, 2007, vol. 1, pp. 165-170.

[28] S. K. Johnson, "3D inline solder paste inspection-Benefit realized," PCB Pulse Article [Online]. Available: http://www.home.agilent.com/ upload/cmc_upload/Al1/SPI_2D_3D_sjohnson_060103.pdf.

Tak-Wai Hui, photograph and biography not available at the time of publication.

Grantham Kwok-Hung Pang, (SM'01) photograph and biography not available at the time of publication. 